\title{
Shadowing in Compton scattering on nuclei
}

\author{
B.Z. Kopeliovich $*$ Ivan Schmidt $]^{\dagger}$ and M. Siddikov \\ Departamento de Física, Centro de Estudios Subatómicos, y Centro Científico - Tecnológico de Valparaíso, \\ Universidad Técnica Federico Santa María, Casilla 110-V, Valparaíso, Chile
}

\begin{abstract}
We evaluate the shadowing effect in deeply virtual and real Compton scattering on nuclei in the framework of the color dipole model. We rely on the soft photon wave function derived in the instanton vacuum model, and employ the impact parameter dependent phenomenological elastic dipole amplitude. Both the effects of quark and the gluon shadowing are taken into account.
\end{abstract}

\section{INTRODUCTION}

Compton scattering, $\gamma^{*}+p \rightarrow \gamma+p$, with initial photons both real or virtual, has been a subject of intensive theoretical and experimental investigation [1 16]. While in the case of deeply-virtual Compton scattering (DVCS), where the initial photon is highly-virtual, the QCD factorization has been proven [5, 7, 8] and the amplitude can be expressed in terms of the generalized parton distributions (GPD) [1-15], in the case of real Compton scattering (RCS) the available theoretical tools are rather undeveloped.

On the one hand, as it has been shown in [17, 18], for large momentum transfer $\Delta_{\perp}$ it is possible to factorize the RCS amplitude [19, 20] and express it in terms of the distribution amplitudes of the proton. On the other hand, it is possible to express the amplitude of the process via the minus 1st-moment of GPDs at zero skewedness [5, 21, 22].

DVCS and RCS on a proton have been studied recently within the color dipole approach in [23, 24]. Here we extend that study to nuclear targets. The DVCS process on a nuclear target has been measured at HERA by HERMES collaboration [26] and may be also studied at the future Electron Ion Collider (EIC) and Large Hadron Electron Collider (LHeC) 27, 28]. The Real Compton Scattering (RCS) may be measured at the LHC, as a subprocess in hadron-hadron collisions in ultraperipheral kinematics. Since both DVCS and RCS are studied in the high-energy kinematics, the nuclear effects reveal themselves as shadowing corrections.

The general framework for evaluation of the shadowing corrections is the Gribov-Glauber approach [29]. While in the asymptotic high-energy ("frozen") regime the shadowing corrections were studied in [25, 30], we use an approach which is also valid for intermediate energies. Also, we take into account the gluon shadowing corrections, which appear for $x_{B} \lesssim 10^{-3}$ and give a sizeable contribution for $x_{B} \sim 10^{-5}$.

The paper is organized as follows. In Sections \we review the general formalism of the color dipole approach. In Section [II we discuss the frequently used frozen approximation which is valid for asymptotically large energies. In Section IV we discuss the method which will be used for calculations of nuclear shadowing effects and demonstrate that for asymptotically large energies it reproduces the results from Section [III In Section $\nabla$ we discuss the gluon shadowing and its effect on the DVCS and RCS observables. In Section VI the wavefunction of a real photon is evaluated in the instanton vacuum model. In Section VII we present the results of numerical evaluation, and in Section VIII we draw conclusions.

\section{COLOR DIPOLE MODEL}

The color dipole model is particularly efficient at high energies, where the dominant contribution to the Compton amplitude comes from gluonic exchanges. Then the general expression for the Compton amplitude on a nucleon has the form,

$$
\mathcal{A}_{\mu \nu}^{(i j)}(s, \Delta)=e_{\mu}^{(i)} e_{\nu}^{(j)} \int_{0}^{1} d \beta_{1} d \beta_{2} d^{2} r_{1} d^{2} r_{2} \bar{\Psi}_{f}^{(i)}\left(\beta_{2}, \vec{r}_{2}\right) \mathcal{A}^{d}\left(\beta_{1}, \vec{r}_{1} ; \beta_{2}, \vec{r}_{2} ; \Delta\right) \Psi_{i n}^{(j)}\left(\beta_{1}, \vec{r}_{1}\right)
$$

\footnotetext{
*Electronic address: Boris.Kopeliovich@usm.cl

${ }^{\dagger}$ Electronic address: Ivan.Schmidt@usm.cl

‡Electronic address: Marat.Siddikov@usm.cl
} 
where $e_{\mu}^{(i)}$ is the photon polarization vector; $\beta_{1,2}$ are the light-cone fractional momenta of the quark and antiquark, $\vec{r}_{1,2}$ are the transverse distances in the final and initial dipoles respectively; $\Delta$ is the momentum transfer in the Compton scattering, $\mathcal{A}^{d}(\ldots)$ is the scattering amplitude of the dipole on the target (proton or nucleus), and $\Psi_{i n(f)}^{(i)}(\beta, \vec{r})$ are the wavefunctions of the initial and final photons in the polarization state $i$ [31].

At high energies in the small angle approximation, $\Delta / \sqrt{s} \ll 1$, the quark separation and fractional momenta $\beta$ are preserved, so,

$$
\begin{aligned}
\mathcal{A}^{d}\left(\beta_{1}, \vec{r}_{1} ; \beta_{2}, \vec{r}_{2} ; Q^{2}, \Delta\right) & \approx \delta\left(\beta_{1}-\beta_{2}\right) \delta\left(\vec{r}_{1}-\vec{r}_{2}\right) \int d^{2} b^{\prime} e^{i \vec{\Delta} \vec{b}^{\prime}} \Im m f_{\bar{q} q}^{N}\left(\vec{r}_{1}, \vec{b}^{\prime}, \beta_{1}\right) \\
\Im m f_{\bar{q} q}^{N}\left(\vec{r}, \vec{b}^{\prime}, \beta\right) & =\frac{1}{12 \pi} \int \frac{d^{2} k d^{2} \Delta}{\left(k+\frac{\Delta}{2}\right)^{2}\left(k-\frac{\Delta}{2}\right)^{2}} \alpha_{s} \mathcal{F}(x, \vec{k}, \vec{\Delta}) e^{i \vec{b}^{\prime} \cdot \vec{\Delta}} \\
& \times\left(e^{-i \beta \vec{r} \cdot\left(\vec{k}-\frac{\vec{\Delta}}{2}\right)}-e^{i(1-\beta) \vec{r} \cdot\left(\vec{k}-\frac{\vec{\Delta}}{2}\right)}\right)\left(e^{i \beta \vec{r} \cdot\left(\vec{k}+\frac{\vec{\Delta}}{2}\right)}-e^{-i(1-\beta) \vec{r} \cdot\left(\vec{k}+\frac{\vec{\Delta}}{2}\right)}\right)
\end{aligned}
$$

where

$$
\begin{aligned}
\frac{\mathcal{F}(x, \vec{k}, \vec{\Delta})}{k^{2}} & \equiv H_{g}(x, \vec{k}, \vec{\Delta})=\frac{1}{2} \int d^{2} r e^{i k \cdot r} \int \frac{d z^{-}}{2 \pi} e^{i x \bar{P}^{+} z^{-}} \times \\
& \times\left\langle P^{\prime}\left|G_{+\alpha}\left(-\frac{z}{2},-\frac{\vec{r}}{2}\right) \gamma_{+} \mathcal{L}\left(-\frac{z}{2}-\frac{\vec{r}}{2}, \frac{z}{2}+\frac{\vec{r}}{2}\right) G^{+\alpha}\left(\frac{z}{2}, \frac{\vec{r}}{2}\right)\right| P\right\rangle
\end{aligned}
$$

is the gluon GPD of the target, $P^{\prime}=P+\Delta, \bar{P}=\left(P+P^{\prime}\right) / 2, G_{\mu \nu}(x)$ is the gluon loop operator, $\mathcal{L}_{\infty}(x, y)$ is the Wilson factor required by gauge covariance. For this GPD we use a gaussian parameterization [55, 56, 66],

$$
\mathcal{F}(x, \vec{k}, \vec{\Delta})=\frac{3 \sigma_{0}(x)}{16 \pi^{2} \alpha_{s}}\left(k+\frac{\Delta}{2}\right)^{2}\left(k-\frac{\Delta}{2}\right)^{2} R_{0}^{2}(x) \exp \left(-\frac{R_{0}^{2}(x)}{4}\left(\vec{k}^{2}+\frac{\vec{\Delta}^{2}}{4}\right)\right) \exp \left(-\frac{1}{2} B(x) \Delta^{2}\right)
$$

where $\sigma_{0}(x), R_{0}^{2}(x), B(x)$ are the free parameters fixed from the DIS and $\pi p$ scattering data. We shall discuss them in more detail in Section VII] The parameterization (5) does not depend on the longitudinal momentum transfer and decreases exponentially as a function of $\Delta^{2}$. Since the parameterization (5) is an effective one and is valid only in the small- $x$ region, we do not assume that it satisfies general requirements, such as positivity [65] and polynomiality [3] constraints.

The prefactor $\left(k+\frac{\Delta}{2}\right)^{2}\left(k-\frac{\Delta}{2}\right)^{2}$ in (5) guarantees convergence of the integrals in the parameterization (2). In the forward limit, the amplitude (2) reduces to the saturated parameterization of the dipole amplitude proposed by Golec-Biernat and Wüsthoff (GBW) [53],

$$
\sigma_{d}(\beta, r)=2 \int d^{2} b^{\prime} \Im m f_{\bar{q} q}^{N}\left(\vec{r}, \vec{b}^{\prime}, \beta\right)=\frac{1}{6 \pi} \int \frac{d^{2} k}{k^{4}} \alpha_{s}\left(k^{2}\right) \mathcal{F}(x, \vec{k}, \overrightarrow{0})=\frac{\sigma_{0}(x)}{2}\left(1-\exp \left(-\frac{r^{2}}{R_{0}(x)}\right)\right)
$$

Generally, the amplitude $f_{\bar{q} q}^{N}(\ldots)$ involves nonperturbative physics, but the asymptotic behaviour for small $r$ is controlled by pQCD [32]:

$$
f_{\bar{q} q}^{N}(\vec{r}, \vec{\Delta}, \beta)_{r \rightarrow 0} \propto r^{2}
$$

up to slowly varying corrections $\sim \ln (r)$.

Calculation of the differential cross section also involves the real part of scattering amplitude, whose relation to the imaginary part is quite straightforward. According to [33], if the $\operatorname{limit} \lim _{s \rightarrow \infty}\left(\frac{\mathcal{I} m f}{s^{\alpha}}\right)$ exists and is finite, then the real and imaginary parts of the forward amplitude are related as

$$
\mathcal{R} e f(\Delta=0)=s^{\alpha} \tan \left[\frac{\pi}{2}\left(\alpha-1+\frac{\partial}{\partial \ln s}\right)\right] \frac{\Im m f(\Delta=0)}{s^{\alpha}} .
$$

In the model under consideration the imaginary part of the forward dipole amplitude indeed has a power dependence on energy, $\mathcal{I} m f(\Delta=0 ; s) \sim s^{\alpha}$, so (7) simplifies to

$$
\frac{\mathcal{R} e \mathcal{A}}{\Im m \mathcal{A}}=\tan \left(\frac{\pi}{2}(\alpha-1)\right) \equiv \epsilon .
$$


This fixes the phase of the forward Compton amplitude, which we retain for nonzero momentum transfers, assuming similar dependences for the real and imaginary parts. Finally we arrive at,

$$
\mathcal{A}_{\mu \nu}^{(i j)}=(\epsilon+i) e_{\mu}^{(i)}\left(q^{\prime}\right) e_{\nu}^{(j)}(q) \int d^{2} r \int_{0}^{1} d \beta \bar{\Psi}_{f}^{(i)}(\beta, r) \Psi_{i n}^{(j)}(\beta, r) \Im m f_{\bar{q} q}^{N}(\vec{r}, \vec{\Delta}, \beta, s),
$$

For the cross-section of unpolarized Compton scattering, from (9) we obtain,

$$
\begin{aligned}
\frac{d \sigma_{e l}^{\gamma p}}{d t} & =\frac{1+\epsilon^{2}}{16 \pi} \sum_{i j}\left|\mathcal{A}_{\mu \nu}^{(i j)}\right|^{2}= \\
& =\frac{1+\epsilon^{2}}{16 \pi} \sum_{i j}\left|\int d^{2} r \int_{0}^{1} d \beta \bar{\Psi}_{f}^{(i)}(\beta, r) \Psi_{i n}^{(j)}(\beta, r) \Im m f_{\bar{q} q}^{N}(\vec{r}, \vec{\Delta}, \beta)\right|^{2}
\end{aligned}
$$

\section{NUCLEAR SHADOWING IN THE "FROZEN" LIMIT}

Nuclear shadowing signals the closeness of the unitarity limit. Hard reactions possess this feature only if they have a contribution from soft interactions. In DIS and DVCS the soft contribution arises from the so called aligned jet configurations [34], corresponding to $\bar{q} q$ fluctuations very asymmetric in sharing the photon momentum, $\beta \ll 1$. Such virtual photon fluctuations, having large transverse separation, are the source of shadowing [40].

Calculation of nuclear shadowing simplifies considerably in the case of long coherence length [35], i.e. long lifetime of the photon fluctuations, when it considerably exceeds the nuclear size. In this case Lorentz time dilation "freezes" the transverse size of the fluctuation during propagation though the nucleus. Then the Compton amplitude of coherent scattering, which leaves the nucleus intact, has the same form as Eq. (9) with a replacement of the nucleon Compton amplitude by the nuclear one,

$$
\Im m f_{\bar{q} q}^{N}(r, \beta, \Delta) \Rightarrow \Im m f_{\bar{q} q}^{A}(r, \beta, \Delta)=\int d^{2} b e^{i \vec{\Delta} \cdot \vec{b}}\left[1-e^{-\Im m f_{\bar{q} q}^{N}(r, \beta, \Delta=0) T_{A}(b)}\right],
$$

where $b$ is impact parameter of the photon-nucleus collision, $T_{A}(b)=\int_{-\infty}^{\infty} d z \rho_{A}(b, z)$ is the nuclear thickness function, given by the integral of nuclear density along the direction of the collisions. In this expression we neglect the real part of the amplitude which is particularly small for a coherent nuclear interaction.

For incoherent Compton scattering, which results in nuclear fragmentation without particle production (quasielastic scattering), the cross section has the form [36],

$$
\begin{aligned}
\frac{d \sigma_{q e l}^{\gamma A}}{d t} & =B_{e l} e^{B_{e l} t} \sum_{i j} \int_{0}^{1} d \beta \int d^{2} r d^{2} r^{\prime} \bar{\Psi}_{f}^{(i)}(\beta, r) \bar{\Psi}_{f}^{(i)}\left(\beta, r^{\prime}\right) \Psi_{i n}^{(j)}(\beta, r) \Psi_{i n}^{(j)}\left(\beta, r^{\prime}\right) \\
& \times \exp \left[\frac{1}{2}\left(\sigma_{\bar{q} q}(r)-\sigma_{\bar{q} q}\left(r^{\prime}\right)\right) T_{A}(b)\right]\left\{\exp \left[\frac{\sigma_{\bar{q} q}(r) \sigma_{\bar{q} q}\left(r^{\prime}\right)}{16 \pi B_{e l}} T_{A}(b)\right]-1\right\} \\
& \approx \frac{e^{B_{e l} t}}{16 \pi} \int d^{2} b T_{A}(b)\left|\int_{0}^{1} d \beta d^{2} r \bar{\Psi}_{f}(\beta, r) \sigma_{\bar{q} q}(r, s) \Psi_{i n}(\beta, r) \exp \left[-\frac{1}{2} \sigma_{\bar{q} q}(r) T_{A}(b]\right)\right|^{2}
\end{aligned}
$$

Here $B_{e l}$ is the $t$-slope of elastic dipole-nucleon amplitude. In this equation we treated the term quadratic in the dipole cross section as a small number and expanded the exponential in curly brackets.

\section{ONSET OF NUCLEAR SHADOWING}

\section{A. Coherent Compton scattering}

The regime of frozen dipole size discussed in the previous section is valid only at very small $x_{B}$ in DVCS, or at high energies in RCS. However, at medium small $x_{B}$ a dipole can "breath", i.e. vary its size, during propagation through the nucleus, and one should rely on a more sophisticated approach. 
In this paper we employ the description of the onset of shadowing developed in [37] and based on the light-cone Green function technique [38]. The propagation of a color dipole in a nuclear medium is described as motion in an absorptive potential, i.e.

$$
i \frac{\partial W\left(z_{2}, r_{2} ; z_{1}, r_{1}\right)}{\partial z_{2}}=-\frac{\Delta_{r_{2}} W\left(z_{2}, r_{2} ; z_{1}, r_{1}\right)}{\nu \alpha(1-\alpha)}-\frac{i \rho_{A}\left(z_{2}, r_{2}\right) \sigma_{\bar{q} q}\left(r_{2}\right)}{2} W\left(z_{2}, r_{2} ; z_{1}, r_{1}\right)
$$

where the Green function $W\left(z_{2}, r_{2} ; z_{1}, r_{1}\right)$ describes the probability amplitude for propagation of dipole state with size $r_{1}$ at the light-cone starting point $z_{1}$ to the dipole state with size $r_{2}$ at the light-cone point $z_{2}$. Then the shadowing correction to the amplitude has the form

$$
\begin{aligned}
\delta A\left(s, \vec{\Delta}_{\perp}\right)= & \int d^{2} b e^{i \vec{\Delta} \cdot \vec{b}_{\perp}} \int_{z_{1} \leq z_{2}} d z_{1} d z_{2} \rho_{A}\left(b, z_{1}\right) \rho_{A}\left(b, z_{2}\right) \int_{0}^{1} d \alpha d^{2} r_{1} d^{2} r_{2} \times \\
& \bar{\Psi}_{f}\left(\alpha, r_{2}\right) \sigma_{\bar{q} q}\left(r_{2}\right) W\left(z_{2}, r_{2} ; z_{1}, r_{1}\right) \sigma_{\bar{q} q}\left(r_{1}\right) \Psi_{i n}\left(\alpha, r_{1}\right) e^{i k_{m i n}\left(z_{2}-z_{1}\right)}
\end{aligned}
$$

where

$$
k_{m i n}=\frac{Q^{2} \alpha(1-\alpha)+m_{q}^{2}}{2 \nu \alpha(1-\alpha)}
$$

Equation (13) is quite complicated and in the general case may be solved only numerically [39]. However in some cases an analytic solution is possible. For example, in the limit of long coherence length, $l_{c} \gg R_{A}$, relevant for high-energy accelerators like LHC, one can neglect the "kinetic" term $\propto \Delta_{r_{2}} W\left(z_{2}, r_{2} ; z_{1}, r_{1}\right)$ in (13), and get the Green function in the "frozen" approximation [38],

$$
W\left(z_{2}, r_{2} ; z_{1}, r_{1}\right)=\delta^{2}\left(r_{2}-r_{1}\right) \exp \left(-\frac{1}{2} \sigma_{\bar{q} q}\left(r_{1}\right) \int_{z_{1}}^{z_{2}} d \zeta \rho_{A}(\zeta, b)\right)
$$

Then the shadowing correction (15) simplifies to

$$
\begin{aligned}
\delta \mathcal{A}\left(s, \Delta_{\perp}\right)= & \int d^{2} b e^{i \vec{\Delta}_{\perp} \cdot \vec{b}_{\perp}} \int_{z_{1} \leq z_{2}} d z_{1} d z_{2} \rho_{A}\left(z_{1}, b\right) \rho_{A}\left(z_{2}, b\right) \int_{0}^{1} d \alpha d^{2} r \sigma_{\bar{q} q}^{2}(r, b) \times \\
& \bar{\Psi}_{f}(\alpha, r) \exp \left(-\frac{1}{2} \sigma_{\bar{q} q}(r) \int_{z_{1}}^{z_{2}} d \zeta \rho_{A}(\zeta, b)\right) \Psi_{i n}(\alpha, r) e^{i k_{m i n}\left(z_{2}-z_{1}\right)} .
\end{aligned}
$$

If we neglect the real part of the amplitude and the longitudinal momentum transfer $k_{\text {min }}$ (which is justified for asymptotically large $s$ ), and average over polarizations, then taking the integral over $z_{1,2}$ "by parts" in (15), we get for the elastic amplitude

$$
\mathcal{A}\left(s, \Delta_{\perp}\right)=2 \int d^{2} b e^{i \vec{\Delta}_{\perp} \cdot \vec{b}_{\perp}} \int_{0}^{1} d \alpha d^{2} r \bar{\Psi}_{f}(\alpha, r)\left[1-\exp \left(-\frac{1}{2} \sigma_{\bar{q} q}(r) \int_{-\infty}^{+\infty} d \zeta \rho_{A}(\zeta, b)\right)\right] \Psi_{i n}(\alpha, r)
$$

Another case where an analytical solution is possible is when the effective dipole sizes are small and the function $\sigma_{\bar{q} q}(r)$ may be approximated as

$$
\sigma_{\bar{q} q}(r) \approx C r^{2}
$$

This approximation cannot be precise even at high virtuality $Q^{2}$ in DVCS, since there are contributions of the aligned jet configurations mentioned above, which permit large dipoles even for large $Q^{2}$. Moreover, such aligned jet configurations of the dipole provide the main contribution to nuclear shadowing [40]. Nevertheless, for the sake of simplicity we rely on this approximation in order to estimate the magnitude of the shadowing corrections. Then Eq. (13) yields for $W\left(z_{2}, r_{2} ; z_{1}, r_{1}\right)$ the well-known evolution operator of harmonic oscillator, although with complex 
frequency

$$
\begin{aligned}
W\left(z_{2}, r_{2} ; z_{1}, r_{1}\right) & =\frac{a}{2 \pi i \sin (\omega \Delta z)} \exp \left(\frac{i a}{2 \sin (\omega \Delta z)}\left[\left(r_{1}^{2}+r_{2}^{2}\right) \cos (\omega \Delta z)-2 \vec{r}_{1} \cdot \vec{r}_{2}\right]\right), \\
\omega^{2} & =\frac{-2 i C \rho_{A}}{\nu \alpha(1-\alpha)}, \\
a^{2} & =-i C \rho_{A} \nu \alpha(1-\alpha) / 2
\end{aligned}
$$

Notice that for DVCS in the kinematics of the HERMES experiment [26] the coherence length

$$
l_{c} \approx \frac{1}{2 m_{N} x_{B}} \sim 1.7 \mathrm{fm}
$$

is comparable with the mean inter-nucleon spacing in nuclei and is much smaller than the radii of heavy nuclei. Therefore the frozen approximation employed in 25] cannot be used for an interpretation of HERMES data, instead one should rely on the Green function method described above.

\section{B. Incoherent scattering}

In addition to the coherent processes which leave the recoil nucleus intact, a large contribution to photon production comes from incoherent Compton scattering, where the target nucleus breaks up. Using the missing mass technique one can select events $\gamma A \rightarrow \gamma A^{*}$ in which the nucleus breaks up to fragments without production of mesons. In this case one can employ completeness of the final states, which greatly simplifies the calculations.

The analysis of such processes in electroproduction of vector mesons was done in [41], and its extension to the DVCS and RCS is quite straightforward and yields

$$
\begin{aligned}
\left.\frac{d \sigma}{d t}\right|_{\Delta_{\perp}=0}= & \frac{1}{16 \pi} \int d^{2} b d z \rho_{A}(b, z)\left|F_{1}(b, z)-F_{2}(b, z)\right|^{2} \\
F_{1}(b, z)= & \int_{0}^{1} d \alpha d^{2} r_{1} d^{2} r_{2} \bar{\Psi}_{f}\left(\alpha, \vec{r}_{2}\right) W\left(+\infty, \vec{r}_{2} ; z, \vec{r}_{1}\right) \sigma_{\bar{q} q}\left(\vec{r}_{1}, s\right) \Psi_{i n}\left(\alpha, \vec{r}_{1}\right) \\
F_{2}(b, z)= & \int_{-\infty}^{z} d z_{2} \int_{0}^{1} d \alpha d^{2} r_{1} d^{2} r_{2} d^{2} r_{3} \bar{\Psi}_{f}\left(\alpha, \vec{r}_{3}\right) W\left(+\infty, \vec{r}_{3} ; z, \vec{r}_{2}\right) \sigma_{\bar{q} q}\left(\vec{r}_{2}, s\right) \times \\
& W\left(z, \vec{r}_{2} ; z_{2}, \vec{r}_{1}\right) \rho_{A}\left(z_{2}, b\right) \sigma_{\bar{q} q}\left(\vec{r}_{1}, s\right) \Psi_{i n}\left(\alpha, \vec{r}_{1}\right) .
\end{aligned}
$$

At sufficiently high energies one can rely on the frozen approximation introduced in the previous section, and this formula may be simplified [42],

$$
\begin{aligned}
F_{1}(b, z)= & \int_{0}^{1} d \alpha d^{2} r \bar{\Psi}_{f}(\alpha, r) \sigma_{\bar{q} q}\left(r_{1}, s\right) \Psi_{i n}(\alpha, r) \exp \left(-\frac{1}{2} \sigma_{\bar{q} q}(r) \int_{z}^{+\infty} d \zeta \rho_{A}(\zeta, b)\right), \\
F_{2}(b, z)= & \int_{0}^{1} d \alpha d^{2} r \bar{\Psi}_{f}(\alpha, r) \times \\
& {\left[\exp \left(-\frac{1}{2} \sigma_{\bar{q} q}(r) \int_{z}^{+\infty} d \zeta \rho_{A}(\zeta, b)\right)-\exp \left(-\frac{1}{2} \sigma_{\bar{q} q}(r) \int_{-\infty}^{+\infty} d \zeta \rho_{A}(\zeta, b)\right)\right] \sigma_{\bar{q} q}(r, s) \Psi_{i n}(\alpha, r), }
\end{aligned}
$$

Correspondingly the cross section (19) takes the form,

$$
\left.\frac{d \sigma}{d t}\right|_{\Delta_{\perp}=0}=\frac{1}{16 \pi} \int d^{2} b T_{A}(b)\left|\int_{0}^{1} d \alpha d^{2} r \bar{\Psi}_{f}(\alpha, r) \sigma_{\bar{q} q}(r, s) \Psi_{i n}(\alpha, r) \exp \left(-\frac{1}{2} \sigma_{\bar{q} q}(r) T_{A}(b)\right)\right|^{2} .
$$


This expression reproduces Eq. (12) derived in the "frozen" limit. It is easy to see that in the limit of a transparent nucleus, $\sigma_{\bar{q} q} T_{A} \ll 1$, the cross section Eq. (24) rises linearly with $A$. However in the limit of a very opaque nucleus (black disk limit), $\sigma_{\bar{q} q} T_{A} \gg 1$, the absorptive exponential factor in (24) terminates the contribution of central impact parameters, and $d \sigma /\left.d t\right|_{\Delta_{\perp}=0} \propto A^{1 / 3}$.

In case of the approximation (17), we may use the explicit expression (18) for the Green function $W\left(z_{2}, \vec{r}_{2} ; z_{1}, \vec{r}_{1}\right)$ to get

$$
\begin{aligned}
F_{1}(b, z) & =\int_{0}^{1} d \alpha d^{2} r_{1} d^{2} r_{2} \bar{\Psi}_{f}\left(\alpha, \vec{r}_{2}\right) \frac{a}{2 \pi i \sin (\omega \Delta z)} \exp \left(\frac{i a}{2 \sin (\omega \Delta z)}\left[\left(r_{1}^{2}+r_{2}^{2}\right) \cos (\omega \Delta z)-2 \vec{r}_{1} \cdot \vec{r}_{2}\right]\right)_{\Delta z=z_{\infty}-z} \\
& \times \sigma_{\bar{q} q}\left(\vec{r}_{1}, s\right) \Psi_{i n}\left(\alpha, \vec{r}_{1}\right), \\
F_{2}(b, z) & =\int_{-\infty}^{z} d z_{2} \int_{0}^{1} d \alpha d^{2} r_{1} d^{2} r_{2} d^{2} r_{3} \bar{\Psi}_{f}\left(\alpha, \vec{r}_{3}\right) \sigma_{\bar{q} q}\left(\vec{r}_{2}, s\right) \rho_{A}\left(b, z_{2}\right) \sigma_{\bar{q} q}\left(\vec{r}_{1}, s\right) \\
& \times \frac{a}{2 \pi i \sin (\omega \Delta z)} \exp \left(\frac{i a}{2 \sin (\omega \Delta z)}\left[\left(r_{3}^{2}+r_{2}^{2}\right) \cos (\omega \Delta z)-2 \vec{r}_{3} \cdot \vec{r}_{2}\right]\right)_{\Delta z=z_{\infty}-z_{2}} \\
& \times \frac{a}{2 \pi i \sin (\omega \Delta z)} \exp \left(\frac{i a}{2 \sin (\omega \Delta z)}\left[\left(r_{1}^{2}+r_{2}^{2}\right) \cos (\omega \Delta z)-2 \vec{r}_{1} \cdot \vec{r}_{2}\right]\right)_{\Delta z=z_{2}-z} \Psi_{i n}\left(\alpha, \vec{r}_{1}\right) .
\end{aligned}
$$

\section{GLUON SHADOWING}

It has been known since 43 that in addition to the quark shadowing inside nuclei there is also a shadowing of gluons, which leads to attenuation of the gluon parton distributions. While nuclear shadowing of quarks is directly measured in DIS, the shadowing of gluons is poorly known from data [44, 45], mainly due to the relatively large error bars in the nuclear structure functions and their weak dependence on the gluon distributions, which only comes via evolution. The theoretical predictions for the gluon shadowing strongly depend on the implemented model-while for $x_{B} \gtrsim 10^{-3}$ they all predict that the gluon shadowing is small, for $x_{B} \lesssim 10^{-3}$ the predictions vary in a wide range (see the review [45] and references therein). Since in this paper we also make predictions for the LHC energy range, the gluon shadowing corrections should be taken into account as well.

In the framework of the color dipole model the gluon attenuation factor $R_{g}$ was evaluated in the Gribov-Glauber approach in [46]. It was found convenient to evaluate the gluon attenuation ratio $R_{g}$ defined as

$$
R_{g}\left(x, Q^{2}, b\right)=\frac{G_{A}\left(x, Q^{2}, b\right)}{T_{A}(b) G_{N}\left(x, Q^{2}, b\right)}
$$

where $G_{N}\left(x, Q^{2}, b\right)$ is the impact parameter dependent gluon GPD, relating it to the shadowing corrections in DIS with longitudinally polarized photons,

$$
R_{g}\left(x, Q^{2}, b\right) \approx 1-\frac{\Delta \sigma_{L}^{\gamma^{*} p}\left(x, Q^{2}, b\right)}{T_{A}(b) \sigma_{L}^{\gamma^{*} p}\left(x, Q^{2}\right)},
$$

where $\Delta \sigma_{L}^{\gamma^{*} p}=\sigma_{L}^{\gamma^{*} A}-A \sigma_{L}^{\gamma^{*} p}$ is the shadowing correction at impact parameter $b$, and $\sigma_{L}^{\gamma^{*} p}\left(x, Q^{2}\right)$ is the total photoabsorption cross section for a longitudinal photon. The process with longitudinal photons is chosen because the aligned jets configurations are suppressed by powers of $Q^{2}$, so that the average size of the dipole is small, $\left\langle r^{2}\right\rangle \sim 1 / Q^{2}$, and nuclear shadowing mainly originates from gluons.

As it was shown in [46],

$$
\Delta \sigma_{L}^{\gamma^{*} p}\left(x, Q^{2}, b\right)=\int_{-\infty}^{+\infty} d z_{1} \int_{-\infty}^{+\infty} d z_{2} \Theta\left(z_{2}-z_{1}\right) \rho_{A}\left(b, z_{1}\right) \rho_{A}\left(b, z_{2}\right) \Gamma\left(x, Q^{2}, z_{2}-z_{1}\right),
$$

where $\rho_{A}(b, z)$ is the nuclear density, and $\Gamma\left(x, Q^{2}, \Delta z\right)$ is defined as

$$
\Gamma\left(x, Q^{2}, \Delta z\right)=\Re e \int_{x}^{0.1} \frac{d \alpha_{G}}{\alpha_{G}} \frac{16 \alpha_{e m}\left(\sum Z_{q}^{2}\right) \alpha_{s}\left(Q^{2}\right) C_{e f f}^{2}}{3 \pi^{2} Q^{2} \tilde{b}^{2}} \times
$$




$$
\begin{aligned}
& \times\left[\left(1-2 \zeta-\zeta^{2}\right) e^{-\zeta}+\zeta^{2}(3+\zeta) E_{1}(\zeta)\right] \\
& \times\left[\frac{t}{w}+\frac{\sinh (\Omega \Delta z)}{t} \ln \left(1-\frac{t^{2}}{u^{2}}\right)+\frac{2 t^{3}}{u w^{2}}+\frac{t \sinh (\Omega \Delta z)}{w^{2}}+\frac{4 t^{3}}{w^{3}}\right]
\end{aligned}
$$

with

$$
\begin{aligned}
\tilde{b}^{2} & =(0.65 G e V)^{2}+\alpha_{G} Q^{2}, \\
\Omega & =\frac{i B}{\alpha_{G}\left(1-\alpha_{G}\right) \nu}, \\
B & =\sqrt{\tilde{b}^{4}-i \alpha_{G}\left(1-\alpha_{G}\right) \nu C_{e f f} \rho_{A}}, \\
\nu & =\frac{Q^{2}}{2 m_{N} x} \\
\zeta & =i x m_{N} \Delta z \\
t & =\frac{B}{\tilde{b}^{2}} \\
u & =t \cosh (\Omega \Delta z)+\sinh (\Omega \Delta z), \\
w & =\left(1+t^{2}\right) \sinh (\Omega \Delta z)+2 t \cosh (\Omega \Delta z),
\end{aligned}
$$

For heavy nuclei we may rely on the hard sphere approximation, $\rho_{A}(r) \approx \rho_{A}(0) \Theta\left(R_{A}-r\right)$, and simplify (28) to:

$$
\Delta \sigma^{\gamma^{*} p}\left(x, Q^{2}, b\right) \approx \rho_{A}^{2}(0) \int_{0}^{L} d \Delta z(L-\Delta z) \Gamma\left(x, Q^{2}, \Delta z\right),
$$

where $L=2 \sqrt{R_{A}^{2}-b^{2}}$. For the total cross-section after integration over $\int d^{2} b$ we may get

$$
\begin{aligned}
\Delta \sigma^{\gamma^{*} p}\left(x, Q^{2}\right) & =\int d^{2} b \Delta \sigma^{\gamma^{*} p}\left(x, Q^{2}, b\right) \\
& \approx \frac{\pi \rho_{A}^{2}(0)}{12} \int_{0}^{2 R} d \Delta z \Gamma\left(x, Q^{2}, \Delta z\right)\left(16 R_{A}^{3}-12 R_{A}^{2} \Delta z+\Delta z^{3}\right)
\end{aligned}
$$

The results of evaluation of the gluon shadowing are presented in Section VII

\section{WAVEFUNCTIONS FROM THE INSTANTON VACUUM}

In this section we present briefly some details of evaluation of the wavefunction in the instanton vacuum model (see [47-49] and references therein). The central object of the model is the effective action for the light quarks in the instanton vacuum, which in the leading order in $N_{c}$ has the form [48, 49]

$$
S=\int d^{4} x\left(\frac{N}{V} \ln \lambda+2 \Phi^{2}(x)-\bar{\psi}\left(\hat{p}+\hat{v}-m-c \bar{L} f \otimes \Phi \cdot \Gamma_{m} \otimes f L\right) \psi\right),
$$

where $\Gamma_{m}$ is one of the matrices, $\Gamma_{m}=1, i \vec{\tau}, \gamma_{5}, i \vec{\tau} \gamma_{5}, \psi$ and $\Phi$ are the fields of constituent quarks and mesons respectively, $N / V$ is the density of the instanton gas, $\hat{v} \equiv v_{\mu} \gamma^{\mu}$ is the external vector current corresponding to the photon, $L$ is the gauge factor,

$$
L(x, z)=P \exp \left(i \int_{z}^{x} d \zeta^{\mu} v_{\mu}(\zeta)\right),
$$

which provides the gauge covariance of the action, and $f(p)$ is the Fourier transform of the zero-mode profile.

In the leading order in $N_{c}$, we have the same Feynman rules as in perturbative theory, but with a momentumdependent quark mass $\mu(p)$ in the quark propagator

$$
S(p)=\frac{1}{\hat{p}-\mu(p)+i 0} .
$$


The mass of the constituent quark has a form

$$
\mu(p)=m+M f^{2}(p),
$$

where $m \approx 5 \mathrm{MeV}$ is the current quark mass, $M \approx 350 \mathrm{MeV}$ is the dynamical mass generated by the interaction with the instanton vacuum background. Due to the presence of the instantons the coupling of a vector current to a quark is also modified,

$$
\begin{aligned}
\hat{v} & \equiv v_{\mu} \gamma^{\mu} \rightarrow \hat{V}=\hat{v}+\hat{V}^{n o n l}, \\
\hat{V}^{n o n l} & \approx-2 M f(p) \frac{d f(p)}{d p_{\mu}} v_{\mu}(q)+\mathcal{O}\left(q^{2}\right) .
\end{aligned}
$$

Notice that for an arbitrary photon momentum $q$ the expression for $\hat{V}^{\text {nonl }}$ depends on the choice of the path in (29) and as a result one can find in the literature different expressions used for evaluations [31, 50 52. In the limit $p \rightarrow \infty$ the function $f(p)$ falls off as $\sim \frac{1}{p^{3}}$, so for large $p \gg \rho^{-1}$, where $\rho \approx(600 \mathrm{MeV})^{-1}$ is the mean instanton size, the mass of the quark $\mu(p) \approx m$ and vector current interaction vertex $\hat{V} \approx \hat{v}$. However we would like to emphasize that the wavefunction $\Psi(\beta, r)$ gets contribution from both the soft and the hard parts, so even in the large- $Q$ limit the instanton vacuum function is different from the well-known perturbative result.

We have to evaluate the wavefunctions associated with the following matrix elements:

$$
I_{\Gamma}(\beta, \vec{r})=\int \frac{d z^{-}}{2 \pi} e^{i\left(\beta+\frac{1}{2}\right) q^{-} z^{+}}\left\langle 0\left|\bar{\psi}\left(-\frac{z}{2} n-\frac{\vec{r}}{2}\right) \Gamma \psi\left(\frac{z}{2} n+\frac{\vec{r}}{2}\right)\right| \gamma(q)\right\rangle,
$$

where $\Gamma$ is one of the matrices $\Gamma=\gamma_{\mu}, \gamma_{\mu} \gamma_{5}, \sigma_{\mu \nu}$. In the leading order in $N_{c}$ one can easily obtain

$$
I_{\Gamma}=\int \frac{d^{4} p}{(2 \pi)^{4}} e^{i \vec{p}_{\perp} \vec{r}_{\perp}} \delta\left(p^{+}-\left(\beta+\frac{1}{2}\right) q^{+}\right) \operatorname{Tr}(S(p) \hat{V} S(p+q) \Gamma) .
$$

The evaluation of (33) is quite tedious but straightforward. Details of this evaluation may be found in [31].

The overlap of the initial and final photon wavefunctions in (10) was evaluated according to

$$
\Psi^{(i) *}\left(\beta, r, Q^{2}=0\right) \Psi^{(i)}\left(\beta, r, Q^{2}\right)=\sum_{\Gamma} I_{\Gamma}^{*}\left(\beta, r^{*}, 0\right) I_{\Gamma}\left(\beta, r, Q^{2}\right),
$$

where summation is done over possible polarization states $\Gamma=\gamma_{\mu}, \gamma_{\mu} \gamma_{5}, \sigma_{\mu \nu}$. In the final state we should use $r_{\mu}^{*}=r_{\mu}+n_{\mu} \frac{q_{\perp}^{\prime} \cdot r_{\perp}}{q_{+}}=r_{\mu}-n_{\mu} \frac{\Delta_{\perp} \cdot r_{\perp}}{q_{+}}$, which is related to the reference frame with $q_{\perp}^{\prime}=0$ in which the components (33) were evaluated.

\section{NUMERICAL RESULTS}

In this section we present the results of numerical calculations. In this paper we consider two processes-DVCS and RCS. While physically they differ only by the kinematics, the parameterizations used for scattering with soft and hard photons are different. For example, for DVCS we have photons with large $Q^{2}$-in this region we have Bjorken scaling, so all the model parameters such as basic cross-section $\sigma_{0}$ and saturation radius $R_{0}$ in Eq. (5). should depend on the Bjorken $x_{B}$. A widely accepted parameterization which incorporates this features is the GBW parameterization [53]. On the contrary, for RCS when $Q^{2}$ is vanishingly small, $x_{B}$ vanishes and thus all variables should depend on Mandelstam $s$. An example of such parameterization is KST parameterization introduced in [54 57]. Up to the best of our knowledge there is no single parameterization which incorporates both asymptotic cases. For this reason for DVCS we used the GBW-style parameterization [53, 66] for the nonintegrated gluon density Eq. (55), which has a form

$$
\begin{aligned}
\sigma_{0}(x) & =23.03 \mathrm{mb}=\mathrm{const}, \\
R_{0}(x) & =0.4 \mathrm{fm} \times\left(x / x_{0}\right)^{0.144}, \\
B(x) & =B_{\gamma^{*} p \rightarrow \rho p}-\frac{1}{8} R_{0}^{2}(x),
\end{aligned}
$$

where $x_{0}=3.04 \times 10^{-4}$, and $B_{\gamma^{*} p \rightarrow \rho p}\left(x, Q^{2} \gg 1 G e V^{2}\right) \approx 5 G e V^{-2}[58$. 

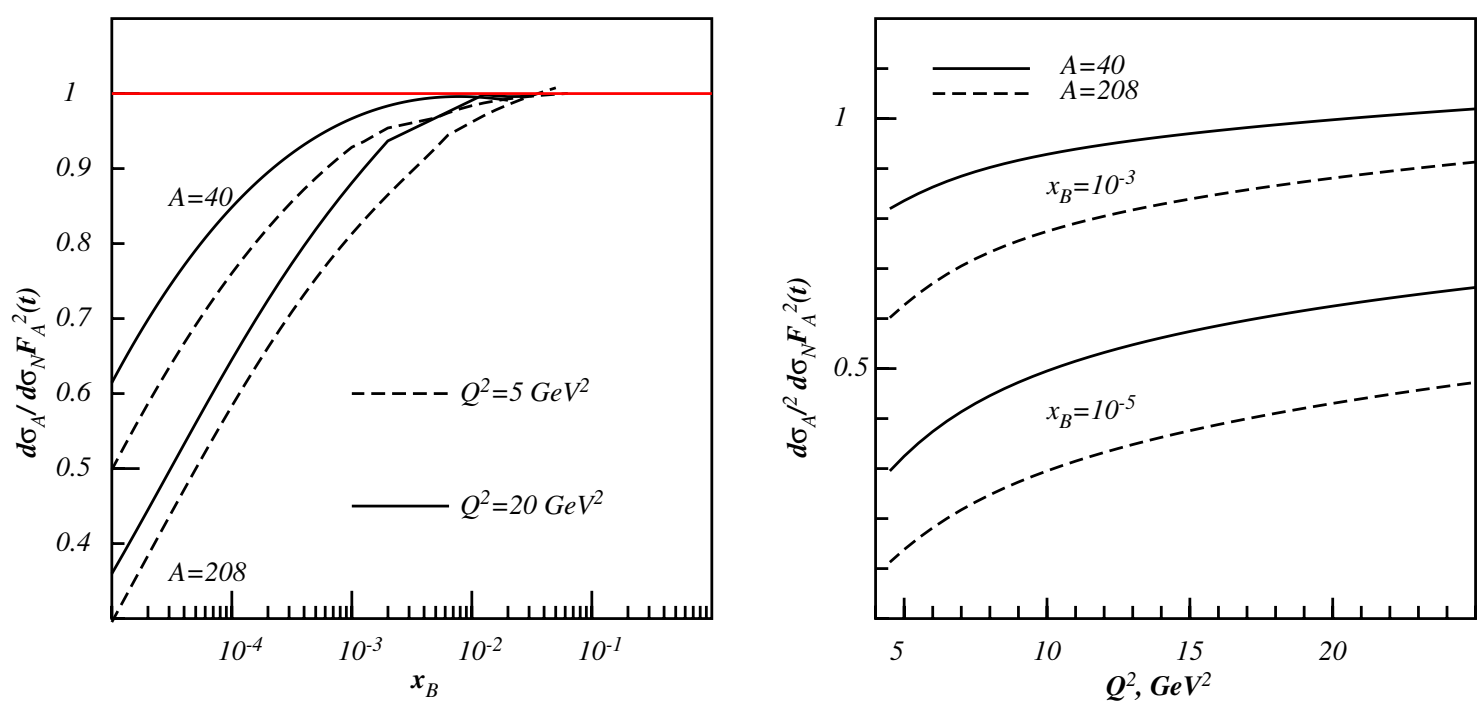

Figure 1: The nucleus to nucleon cross section ratio for coherent DVCS as function of different kinematical variables. Left: $x_{B}$-dependence of the shadowing, $t=t_{\text {min }}$, for different $Q^{2}$ and $A$. From bottom to top: $Q^{2}=5 \mathrm{GeV}^{2}$ and $Q^{2}=20 \mathrm{GeV}^{2}$. Right: $Q^{2}$-dependence, $t=-0.01 \mathrm{GeV}^{2}$, for different $x_{B}$ and $A$. From bottom to top: $x_{B}=10^{-5}$ and $x_{B}=10^{-3}$.

For RCS we used a KST-style parameterization, which has the form [23, 24, 54 [57, 66].

$$
\begin{aligned}
\sigma_{0}(s) & =\sigma_{\pi p}(s)\left(1+\frac{3}{8} \frac{R_{0}^{2}(s)}{r_{\pi}^{2}}\right) \\
\sigma_{\pi p}(s) & =\left(23.6\left(\frac{s}{s_{0}}\right)^{0.079}+1.45\left(\frac{s_{0}}{s}\right)^{0.45}\right) m b \\
R_{0}(s) & =0.88 \mathrm{fm}\left(\frac{s_{0}}{s+s_{1}}\right)^{2} \\
B(s) & =B_{\gamma^{*} p \rightarrow \rho p}-\frac{1}{8} R_{0}^{2}(s)
\end{aligned}
$$

where $s_{0} \approx 1000 \mathrm{GeV}^{2}, s_{1} \approx 3600 \mathrm{GeV}^{2}$. Note that in the large- $s$ region considered in this paper we may neglect the second (Reggeon) term in Eq. (39) and set $s_{1} \approx 0$ in (40). As one can see from the Figure 1, the shadowing correction is increasing towards small $x_{B}$, and for $x_{B} \sim 10^{-5}$ the nuclear cross-section ratio decreases by a factor of two compared to the naive estimate $d \sigma_{A} \sim F_{A}^{2}(t) d \sigma_{N}$. As a function of the momentum transfer $t$, the shadowing correction reveals the behavior qualitatively similar to the nuclear formfactor $F_{A}(t)$ : it steeply drops at small- $t$ and has zeros for some $t$. Notice, however, that the zero positions in the cross-section do not coincide with the zeros of the formfactor. This is a result of shadowing which suppresses the contribution of the central part of the nucleus and modifies the $b$-dependence of the cross section compared to the formfactor. Notice that, as was discussed in [59], if we had sufficiently high resolution in $t$, it would be possible to measure the contributions of the "pure" DVCS, without contributions from the Bethe-Heitler, near the zeros of the formfactor.

The $Q^{2}$-dependence of the nuclear ratio is shown in the right pane of the Figure 1 .

In the Figure 2 we compare the results for the coherent cross section ratio evaluated with and without gluon shadowing. As expected, gluon shadowing is very small at $x_{B} \gtrsim 10^{-3}$ and gives $\sim 20-30 \%$ contribution for $x \sim 10^{-5}$. Similar dependence is observed for all the other cross-sections. In what follows, for the sake of brevity we present only results which include gluon shadowing.

The nuclear ratio for incoherent scattering, $\gamma^{*} A \rightarrow \gamma A^{\prime}$, is shown in the Figure 3 . It turns out that the effect of shadowing at small $x_{B}$ is twice as strong as for the coherent case. As a function of $t$, the nuclear cross-section is almost a constant-due to the nucleus breakup the nuclear cross-section is not suppressed by $F_{A}(t)$ and decreases only as a function of the nucleon formfactor $F_{N}(t)$. The $Q^{2}$ dependence of the nuclear ratio is quite similar to what was found for the coherent case.

For coherent RCS, $\gamma A \rightarrow \gamma A$, the results of evaluation are shown in Figure 4 Similar to DVCS, the cross-section is 

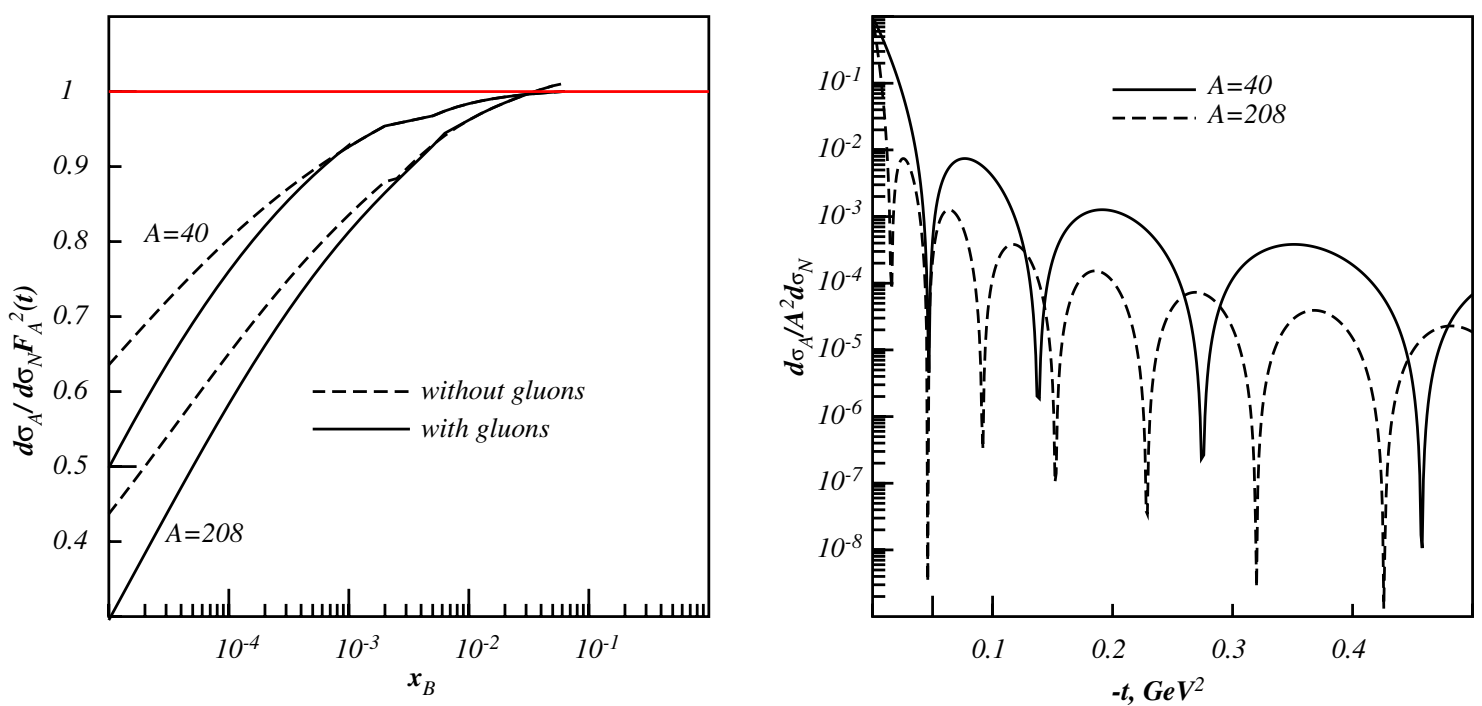

Figure 2: The nucleus to nucleon cross section ratio for coherent DVCS as function of different kinematical variables. Left: shadowing as function of Bjorken $x_{B}$ with and without gluon shadowing for different nuclei, $t=t_{\text {min }}, Q^{2}=5 \mathrm{GeV}^{2}$. Right: $t$-dependence for different $A . x_{B}=10^{-3}, Q^{2}=5 \mathrm{GeV}^{2}$.
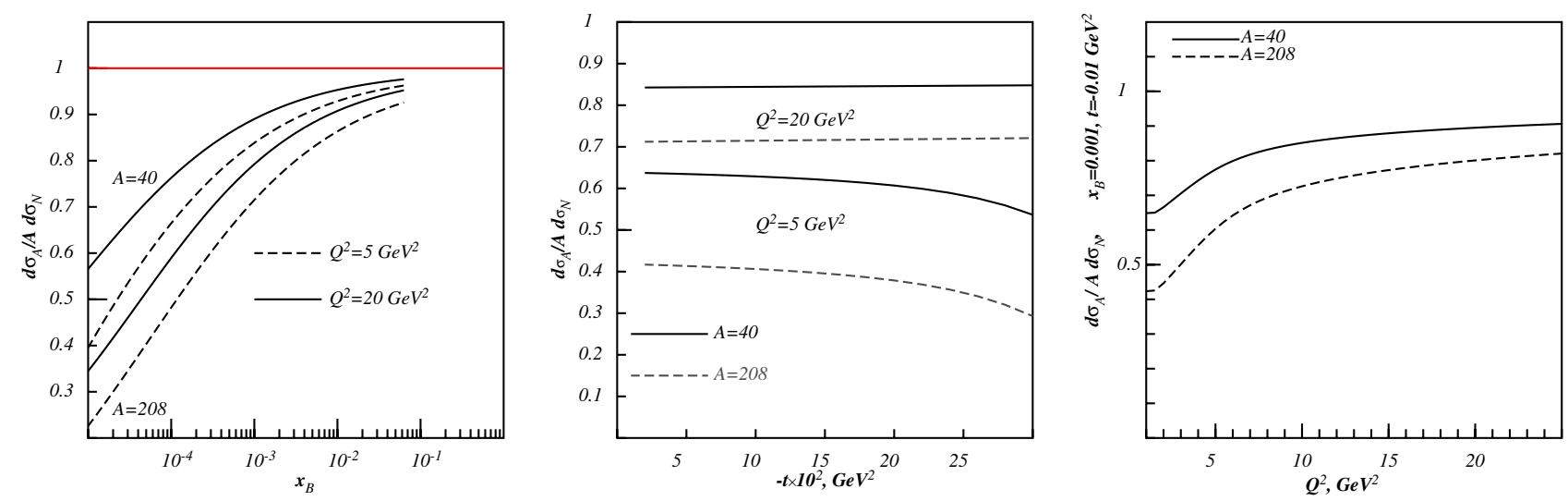

Figure 3: Dependence of the nuclear ratio for incoherent DVCS on different kinematical variables. Left: $x_{B}$-dependence, center: $t$-dependence, right: $Q^{2}$-dependence.

steeply falling with center of mass energy $W$ (for DVCS $x_{B} \propto 1 / W^{2}$ at fixed $\left(Q^{2}, t\right)$ ). As function of the momentum transfer $t$, the shadowing correction reveals the behavior qualitatively similar to the case of coherent DVCS (see Fig. 1) It steeply decreases similar to the behavior of the nuclear formfactor $F_{A}(t)$.

For the incoherent RCS, $\gamma A \rightarrow \gamma A^{\prime}$, the results of evaluation are shown in Figure 5. Similar to the coherent case, the cross-section is decreasing as function of $W$, down to the values an order of magnitude smaller than would give a simple sum of the nucleon cross-sections. As function of $t$, the cross-section is almost constant because of absence of the nuclear formfactor.

\section{CONCLUSIONS AND PROSPECTS}

In this paper we considered DVCS and RCS on nuclear targets within the color dipole model. Results for the coherent shadowing are presented in Section VII. We found that the magnitude of nuclear shadowing is large and is important for analysis of DVCS and RCS data. It may cause a substantial suppression of the nuclear DVCS crosssection. We observed that for incoherent case, the shadowing is stronger than for the coherent case. This happens 

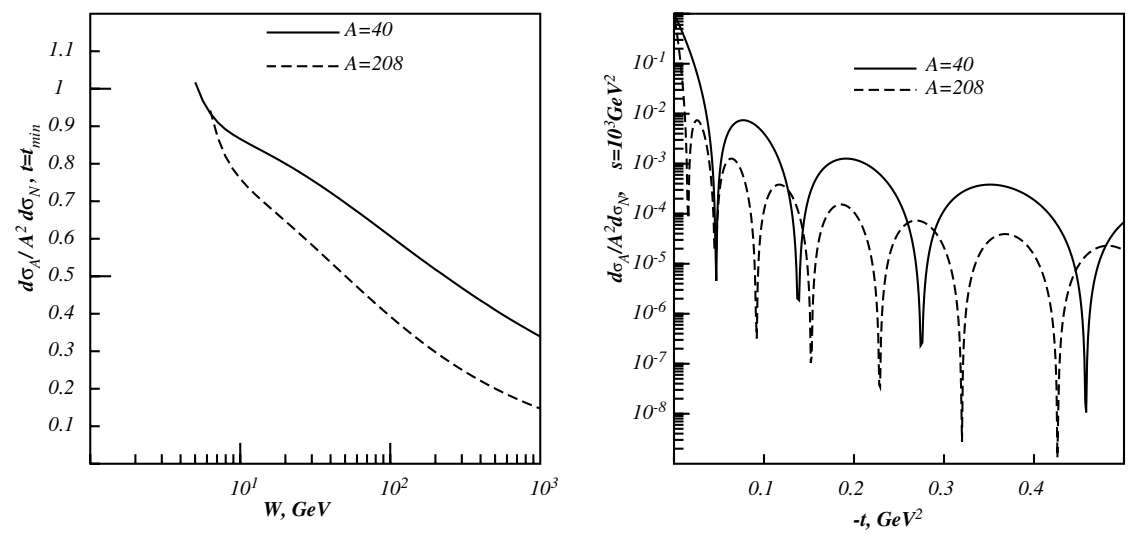

Figure 4: Nuclear ratio for coherent RCS as function of $W$ (left) and $t$ (right).
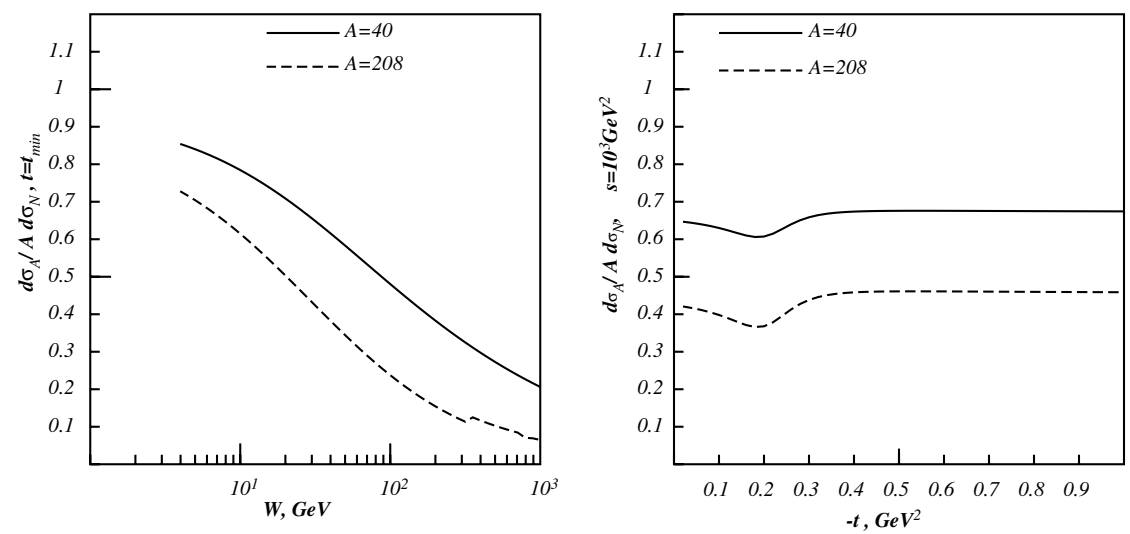

Figure 5: Dependence of the incoherent nuclear RCS cross-section ratio on $W$ (left) and $t$ (right).

since the incoherent cross section is proportional to the survival probability of the dipole, so it vanishes in the black disk limit $\left(\propto A^{1 / 3}\right)$. At the same time, the coherent cross section saturates at a value $\propto A^{2 / 3}$. For the RCS, the shadowing is larger than for the DVCS, since a real photon fluctuates to dipoles of a larger size than a virtual one.

Our results for DVCS and RCS can be applied to $e A$ collisions at future electron-ion colliders (EIC and LHeC, 27, [28]). Currently data for nuclear DVCS are available from the HERMES experiment at HERA [26], however the values of Bjorken $x_{B}$ are too large to produce any sizable shadowing effects. Besides electron beams, the usual source of quasi-real photons, one can also use beams of charged hadrons. Provided that the transverse overlap of the colliding hadrons is small, i.e. the transverse distance $b$ between the centers of the colliding particles exceeds the sum of their radii, $b>R_{1}+R_{2}$, the electromagnetic interaction between colliding particles becomes the dominant mechanism. Such processes called ultra-peripheral collisions (UPC) can be studied in $p p, p A$ and $A A$ collisions. In particular, one can access RCS in the reaction

$$
A_{1}+A_{2} \rightarrow A_{1}+\gamma+A_{2} .
$$

The typical virtualities $\left\langle Q_{\gamma^{*}}^{2}\right\rangle$ of the intermediate photon $\gamma^{*}$ are controlled by the formfactors of the colliding particles, and are small:

$$
\left\langle Q_{\gamma^{*}}^{2}\right\rangle \lesssim \frac{3}{R_{A}^{2}} \sim \frac{0.1 G e V^{2}}{A^{2 / 3}}
$$

where $A$ is the atomic number of the hadron ( $A=1$ for a proton) interacting electromagnetically, i.e. emitting the photon, while the second hadron interacts strongly.

Thus, $\left\langle Q_{\gamma^{*}}^{2}\right\rangle$ is of the order of the soft hadronic scale, so the intermediate photon can be treated as a free WeizsckerWilliams one, i.e. the amplitude of the process (42) can be described in terms of RCS. 
These processes at the LHC will allow to study RCS at very high energies. The possibility of observation of such processes experimentally has been demonstrated by the STAR 60 62] and PHENIX [63] experiments at RHIC. It is expected that at LHC photon-proton collisions at energies up to $\sqrt{s_{\gamma p}} \lesssim 8 \times 10^{3} \mathrm{GeV}$ can be observed [64].

\section{Acknowledgments}

This work was supported in part by Fondecyt (Chile) grants 1090291, 1090073, 1100287, and by DFG (Germany) grant PI182/3-1.

[1] D. Mueller, D. Robaschik, B. Geyer, F. M. Dittes and J. Horejsi, Fortsch. Phys. 42, 101 (1994) [arXiv:hep-ph/9812448].

[2] X. D. Ji, Phys. Rev. D 55, 7114 (1997).

[3] X. D. Ji, J. Phys. G 24, 1181 (1998) [arXiv:hep-ph/9807358].

[4] A. V. Radyushkin, Phys. Lett. B 380, 417 (1996) [arXiv:hep-ph/9604317].

[5] A. V. Radyushkin, Phys. Rev. D 56, 5524 (1997).

[6] A. V. Radyushkin, arXiv:hep-ph/0101225.

[7] X. D. Ji and J. Osborne, Phys. Rev. D 58 (1998) 094018 [arXiv:hep-ph/9801260].

[8] J. C. Collins and A. Freund, Phys. Rev. D 59 (1999) 074009 [arXiv:hep-ph/9801262].

[9] J. C. Collins, L. Frankfurt and M. Strikman, Phys. Rev. D 56, 2982 (1997).

[10] S. J. Brodsky, L. Frankfurt, J. F. Gunion, A. H. Mueller and M. Strikman, Phys. Rev. D 50, 3134 (1994).

[11] K. Goeke, M. V. Polyakov and M. Vanderhaeghen, Prog. Part. Nucl. Phys. 47, 401 (2001) [arXiv:hep-ph/0106012].

[12] M. Diehl, T. Feldmann, R. Jakob and P. Kroll, Nucl. Phys. B 596, 33 (2001) [Erratum-ibid. B 605, 647 (2001)] [arXiv:hep$\mathrm{ph} / 0009255]$.

[13] A. V. Belitsky, D. Mueller and A. Kirchner, Nucl. Phys. B 629, 323 (2002) [arXiv:hep-ph/0112108].

[14] M. Diehl, Phys. Rept. 388, 41 (2003) [arXiv:hep-ph/0307382].

[15] A. V. Belitsky and A. V. Radyushkin, Phys. Rept. 418, 1 (2005) [arXiv:hep-ph/0504030].

[16] V. P. Goncalves, M. V. T. Machado and A. R. Meneses, arXiv:1003.0828 [hep-ph].

[17] A. S. Kronfeld and B. Nizic, Phys. Rev. D 44 (1991) 3445 [Erratum-ibid. D 46 (1992) 2272].

[18] T. C. Brooks and L. J. Dixon, Phys. Rev. D 62 (2000) 114021 [arXiv:hep-ph/0004143].

[19] G. P. Lepage and S. J. Brodsky, Phys. Lett. B 87 (1979) 359.

[20] G. P. Lepage and S. J. Brodsky, Phys. Rev. D 22 (1980) 2157.

[21] M. Diehl, T. Feldmann, R. Jakob and P. Kroll, Eur. Phys. J. C 8 (1999) 409 [arXiv:hep-ph/9811253].

[22] M. Diehl, T. Feldmann, R. Jakob and P. Kroll, Eur. Phys. J. C 39 (2005) 1 [arXiv:hep-ph/0408173].

[23] B. Z. Kopeliovich, I. Schmidt and M. Siddikov, Phys. Rev. D 79 (2009) 034019 [arXiv:0812.3992 [hep-ph]].

[24] B. Z. Kopeliovich, I. Schmidt and M. Siddikov, Phys. Rev. D 80 (2009) 054005 [arXiv:0906.5589 [hep-ph]].

[25] M. V. T. Machado, Eur. Phys. J. C 59 (2009) 769 [arXiv:0810.3665 [hep-ph]].

[26] A. Airapetian et al. [The HERMES collaboration], arXiv:0911.0091 [hep-ex].

[27] M. Klein et al., In the Proceedings of 11th European Particle Accelerator Conference (EPAC 08), Magazzini del Cotone, Genoa, Italy, 23-27 Jun 2008, pp WEOAG01.

[28] F. Zimmermann et al., In the Proceedings of 11th European Particle Accelerator Conference (EPAC 08), Magazzini del Cotone, Genoa, Italy, 23-27 Jun 2008, pp WEPP154.

[29] V. N. Gribov, Sov. Phys. JETP 29 (1969) 483 [Zh. Eksp. Teor. Fiz. 56 (1969) 892].

[30] M. V. T. Machado, arXiv:0905.4516 [hep-ph].

[31] A. E. Dorokhov, W. Broniowski and E. Ruiz Arriola, Phys. Rev. D 74 (2006) 054023 [arXiv:hep-ph/0607171].

[32] B. Z. Kopeliovich, L. I. Lapidus and A. B. Zamolodchikov, JETP Lett. 33 (1981) 595 [Pisma Zh. Eksp. Teor. Fiz. 33 (1981) 612].

[33] J. B. Bronzan, G. L. Kane and U. P. Sukhatme, Phys. Lett. B 49 (1974) 272.

[34] J. D. Bjorken and J. B. Kogut, Phys. Rev. D 8, 1341 (1973).

[35] B. Z. Kopeliovich, J. Raufeisen and A. V. Tarasov, Phys. Rev. C 62, 035204 (2000).

[36] B. Z. Kopeliovich, I. K. Potashnikova and I. Schmidt, Phys. Rev. C 73, 034901 (2006).

[37] B. Z. Kopeliovich, J. Raufeisen and A. V. Tarasov, 440 (1998) 151 [arXiv:hep-ph/9807211].

[38] B. Z. Kopeliovich and B. G. Zakharov, Phys. Rev. D 44, 3466 (1991).

[39] J. Nemchik, Phys. Rev. C 68, 035206 (2003) [arXiv:hep-ph/0301043].

[40] B. Kopeliovich and B. Povh, Phys. Lett. B 367, 329 (1996); Z. Phys. A 356, 467 (1997) [arXiv:nucl-th/9607035].

[41] B. Z. Kopeliovich, J. Nemchik, A. Schafer and A. V. Tarasov, Phys. Rev. C 65 (2002) 035201 [arXiv:hep-ph/0107227].

[42] J. Hufner, B. Kopeliovich and A. B. Zamolodchikov, Z. Phys. A 357, 113 (1997) [arXiv:nucl-th/9607033].

[43] O. V. Kancheli, Pisma Zh. Eksp. Teor. Fiz. 18 (1973) 465.

[44] F. Arleo and T. Gousset, Phys. Lett. B 660 (2008) 181 [arXiv:0707.2944 [hep-ph]].

[45] N. Armesto, 32 (2006) R367 [arXiv:hep-ph/0604108]. 
[46] B. Z. Kopeliovich, J. Raufeisen, A. V. Tarasov and M. B. Johnson, Phys. Rev. C 67 (2003) 014903 [arXiv:hep-ph/0110221].

[47] T. Schafer and E. V. Shuryak, Rev. Mod. Phys. 70 (1998) 323 [arXiv:hep-ph/9610451].

[48] D. Diakonov and V. Y. Petrov, Nucl. Phys. B 272 (1986) 457

[49] D. Diakonov, M. V. Polyakov and C. Weiss, Nucl. Phys. B 461 (1996) 539 [arXiv:hep-ph/9510232].

[50] I. V. Anikin, A. E. Dorokhov and L. Tomio, Phys. Part. Nucl. 31 (2000) 509 [Fiz. Elem. Chast. Atom. Yadra 31 (2000) 1023].

[51] A. E. Dorokhov and W. Broniowski, Eur. Phys. J. C 32 (2003) 79 [arXiv:hep-ph/0305037].

[52] K. Goeke, M. M. Musakhanov and M. Siddikov, Phys. Rev. D 76 (2007) 076007 [arXiv:0707.1997 [hep-ph]]

[53] K. J. Golec-Biernat and M. Wüsthoff, Phys. Rev. D 59 (1999) 014017 [arXiv:hep-ph/9807513].

[54] B.Z. Kopeliovich, A. Schäfer and A.V. Tarasov, Phys. Rev. D62, 054022 (2000).

[55] B. Z. Kopeliovich, H. J. Pirner, A. H. Rezaeian and I. Schmidt, Phys. Rev. D 77 (2008) 034011 [arXiv:0711.3010 [hep-ph]].

[56] B. Z. Kopeliovich, A. H. Rezaeian and I. Schmidt, arXiv:0809.4327 [hep-ph], to appear in Phys. Rev. D.

[57] B. Z. Kopeliovich, J. Nemchik and I. Schmidt, Phys. Rev. C 76 (2007) 025210 [arXiv:hep-ph/0703118].

[58] S. Chekanov et al., (ZEUS Collaboration), PMC Phys. A1, 6 (2007) [arXiv:0708.1478 ].

[59] K. Goeke, V. Guzey and M. Siddikov, Phys. Rev. C 79 (2009) 035210 [arXiv:0901.4711 [hep-ph]].

[60] B. I. Abelev et al. [STAR Collaboration], Phys. Rev. C 77 (2008) 034910 [arXiv:0712.3320 [nucl-ex]].

[61] J. Adams et al. [STAR Collaboration], Phys. Rev. C 70 (2004) 031902 [arXiv:nucl-ex/0404012].

[62] C. Adler et al. [STAR Collaboration], Phys. Rev. Lett. 89 (2002) 272302 [arXiv:nucl-ex/0206004].

[63] D. G. d'Enterria, arXiv:nucl-ex/0601001.

[64] K. Hencken et al., Phys. Rept. 458 (2008) 1 [arXiv:0706.3356 [nucl-ex]].

[65] P. V. Pobylitsa, Phys. Rev. D 65 (2002) 114015 [arXiv:hep-ph/0201030].

[66] B. Z. Kopeliovich, I. K. Potashnikova, I. Schmidt and J. Soffer, Phys. Rev. D 78 (2008) 014031 [arXiv:0805.4534 [hep-ph]]. 